\title{
Mathematical Oncology
}

\author{
Alexander R. A. Anderson $^{1}(\mathbb{D})$ - Philip K. Maini $^{2}$
}

Published online: 20 April 2018

(C) Society for Mathematical Biology 2018

Despite a huge amount of research effort, cancer continues to be a major killer. One of the main reasons for this is the immense complexity of the disease. Cancer is a multiscale process in which genetic mutations occurring at a subcellular level manifest themselves as functional changes at the cellular and tissue scale. Conversely, tissue level properties, such as blood flow, produce Darwinian selection forces that govern the local distribution of cellular phenotypes and genotypes. Changes in tissue lead to a disruption of organ form and function that can ultimately lead to failure and death (Fig. 1). This multiscale aspect of cancer has largely been neglected in the reductionist paradigm, which typically views cancer as "a disease of the genes". The reason for this is clear as the remarkable advances in molecular technology have greatly enhanced quantitative measurements at the genetic scale, while cellular- or tissue-scale properties remain the province of pathology and radiology, which generally lack such tools. The genomic revolution has led to the development of an extensive set of tools to measure and analyze gene expression and mutation. However, the mapping of these changes to in vivo structure and function of cancer cells and tissue remains limited. Furthermore, while tumor evolution is often viewed as a mutation-driven process, it is

Alexander R. A. Anderson and Philip K. Maini have contributed equally.

$\triangle \quad$ Alexander R. A. Anderson

Alexander.Anderson@moffitt.org

$\bowtie \quad$ Philip K. Maini

maini@maths.ox.ac.uk

1 Integrated Mathematical Oncology Department, H. Lee Moffitt Cancer Center and Research Institute, Tampa, FL, USA

2 Wolfson Centre for Mathematical Biology, Mathematical Institute, Oxford, UK 


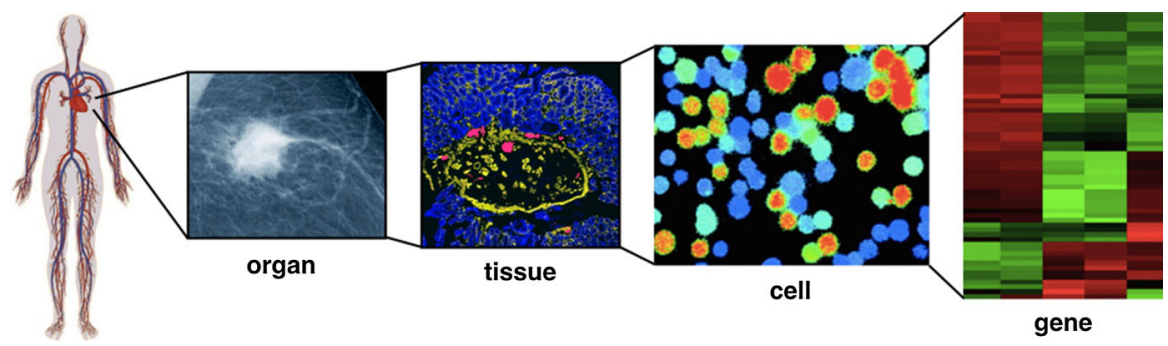

Fig. 1 Cancer is multiscale. Genetic and epigenetic changes lead to modified signaling and changes in cell behavior, giving rise to cancerous tissue impacting organ function and eventually the organism (figure courtesy of Jill Gallaher)

important to recognize that the cell phenotype is the fundamental unit of selection and its fitness is determined through complex interactions with local microenvironmental properties and competing populations.

Tumors are not simply collections of mutated cells that grow in isolation, but rather they are part of a larger organ ecosystem and are actively competing with, and disrupting, a complex community of many interacting cellular and microenvironmental elements that are attempting to maintain homeostasis (Basanta and Anderson 2017). Interactions are really at the heart of an ecological view of cancer, and it is commonly assumed that in an ecosystem with limited resources, nutrients and space for growth, the only interaction among individuals is competition (Maley et al. 2017). However, other ecological interactions such as predation, parasitism, mutualism and commensalism can also be observed. The importance of the microenvironment in cancer progression is now widely recognized (Egeblad et al. 2010). The disruption of organ homeostasis leads to significant microenvironmental heterogeneity. Therefore, heterogeneity is both a cause and consequence of the dialog between the tumor and its environment and has been observed across genotypic (Greaves and Maley 2012; Burrell et al. 2013), phenotypic (Swanton 2012; Meacham and Morrison 2013; Robertson-Tessi et al. 2015) and environmental scales (Tredan et al. 2007; Gatenby and Gillies 2008; Friedl and Alexander 2011) and has now been recognized as a key driver in cancer drug resistance and treatment failure (Meads et al. 2009; Marusyk et al. 2012; Turner and Reis-Filho 2012; Hirata et al. 2015). Ultimately, these complex interactions of tumor cells, environment and therapy produce multiscale heterogeneity in which local microenvironmental conditions select phenotypic tumor clones that are best adapted to locally survive and proliferate and, conversely, the phenotypic properties of the cells affect the environment. This dynamic feedback can only truly be understood through the integration of experiments and mathematical modeling and has driven much of the current field of mathematical oncology. However, if we hope to translate this understanding to the clinic and directly impact patient lives, then these models also need to integrate patient data. We argue that for the next generation of predictive mathematical models to be successful we need the right data.

The big data revolution is in full swing and has been a significant driver of advances in the field of systems biology for the obvious reason that much of it is from one scale: genomic. Genome scale data can direct the choice of specific cancer drugs and potentially classify patients into different categories, and has had many successes, not 
only for cancer (see, for example, Price et al. 2015). However, it is mostly utilized in a correlative manner and thus is only part of the story. In order to tackle major issues, such as treatment resistance, we need causation, or mechanism. The huge recent advances in computational power mean that we can now propose mechanistic mathematical models, but these have to eventually be calibrated through data gathered from patients. The big data issue in cancer, is that for patients, much of the data are not appropriate for such models and, therefore, limit our ability to develop a mechanistic understanding. Specifically, often the data are not longitudinal, not spatial, only from one scale, averaged and homogeneous, not correlated or co-registered and not within an appropriate context. What we need are the "right" data that will allow us to better define the cancer system and connect the scales of cancer, bridging genotype to phenotype, cell to tissue, organ to patient and individual to population. This will require a paradigm shift in how we gather and view patient data, but the potential benefits are enormous. Just by collecting the right data, we will immediately uncover relationships that were not previously seen or understood (for free). Mining these data will provide an even richer and more comprehensive understanding of the relationships across scales and over time. But because of the complex and dynamic nature of cancer, to fully exploit the information in these data and reveal deeper insights will require mathematical and computational models. Moreover, the complexity is such that realistically we need to focus on subsets of the myriad of processes involved, so as to systematically build up a mechanistic understanding of cancer growth and development.

\section{Mathematical Oncology Past, Present and Future}

During the past 15 years, mathematical models of cancer have seen a significant increase in both their number and impact on cancer research (Gatenby and Maini 2003; Anderson and Quaranta 2008; Byrne 2010; Altrock et al. 2015). This is partly due to increasing interest from the oncology community, who have begun to realize the potential mathematical models hold for delivering a more mechanistic understanding of such a complex system. Experimental biologists have also realized that, while reductionist approaches have delivered a great deal, interactions between the individual components are equally, if not more, important for understanding the system. This more systemic view of cancer is naturally a place where mathematical models shine as they readily integrate multiple processes across different biological and temporal scales and capture the complex interactions between cells and their environment.

The above is true in nearly all areas of mathematical biology, and the field is changing so much as to be almost unrecognizable from say 40 years ago. Now, we see models that are rooted much more deeply in the experimental literature and the focus is not only on making experimentally testable predictions, but also testing them with data and, increasingly, actually working with experimentalists to design studies to test model predictions. The process of doing this leads to many challenges and therefore important advances in experimental techniques, mathematical modeling and analysis. In this special issue on Mathematical Oncology, we focus on the latter. The papers in this issue not only show how standard mathematical approaches can be used to generate new biological insights, but also how problem-driven studies naturally 
lead to the development of new modeling techniques, and therefore models that are novel mathematically. Moreover, it becomes clear that the problems presented by biology require methods and techniques that not only come from traditional applied mathematics, but also pure mathematics, statistics and computation.

As we move from models that are focused more on understanding basic biological processes to those that will directly impact patient lives, model predictions need to be more robust and therefore consistent across a suite of models before they can be implemented clinically — we see direct examples of this in our special issue. In addition, we need to embark on more integrated studies where experiments are designed specifically to facilitate the model validation process. This typically will require several rounds of the predict-test-refine-predict cycle before it is safe to use model predictions in a clinical setting.

The future of our field looks incredibly promising, as mechanistic models become more important for not only driving hypothesis generation, but also hypothesis testing. The following represent specific areas where we see the future of mathematical oncology having the largest impact:

(1) Predicting and optimizing patient-specific treatment strategies

(2) Integration of molecular scale data, in a functional manner, into multiscale models

(3) Defining and facilitating the role of the immune system in cancer

(4) Facilitating and augmenting clinical trial design

(5) Understanding and minimizing the emergence of treatment resistance

(6) Greater integration with systems biology.

\section{Special Issue on Mathematical Oncology}

The field of mathematical oncology has been steadily growing for the last decade and now covers the gamut of disease stage, site and process, so the choice of authors for a special issue is bewildering. To narrow down the choice in a rational way, we decided to focus on the emphasis year on Cancer And its Environment, run at the Mathematical Biosciences Institute (MBI), The Ohio State University in 2014-2015, and encompassing many leaders in the field. This year-long program covered many aspects of cancer initiation, progression and treatment. It consisted of seven workshops, with each workshop focusing on different aspects of the disease: (I) Ecology and Evolution of Cancer; (II) Metastasis and Angiogenesis; (III) Cancer and the Immune System; (IV) Tumor Heterogeneity and the Microenvironment; (V) Treatment, Clinical Trials, Resistance; (VI) Targeting Cancer Cell Proliferation and Metabolism Networks; (VII) Stem Cells, Development, and Cancer. This year exemplified the integrated view of mathematical oncology, and we invited the organizers of the workshops in this program to submit their research for this special issue.

The first two papers investigate different facets of angiogenesis. Gravenmier et al. (2017) investigate the potential consequences arising from the fact that the immaturity of tumor vasculature leads to disordered, complex, stochastic flow dynamics to which cells must adapt. They hypothesize that the Warburg effect is actually an adaptation for tumor cells as a bet-hedging strategy. They develop a discrete-time competition model of Lotka-Volterra type and investigate how stochastically fluctuating blood flow could 
serve as an agent for a Darwinian selection pressure due to the resultant disordered oxygen concentrations experienced by cells.

Jain and Jackson (2017) present an ordinary differential equation (ODE) model to investigate the interaction of VEGF, its receptors and intracellular signaling pathways with the aim to understand the effects of VEGF-targeted therapies. Their detailed investigation of the model reveals the danger of using in vitro results to generate in vivo treatments as model predictions show that the results of treatments in one setting are not necessarily reflected in other settings. They show that the model has the potential to help us understand why such therapies may be successful in some cases but not in others. This paper highlights a very important point- the vast majority of experimental systems are also models and, as such, results from such studies should be treated with the same caution as those from a mathematical model. The paper shows how mathematical modeling can help to bridge the gap between in vitro experimental models and in vivo reality.

The next two papers investigate metastasis. Giverso et al. (2017) address mechanical issues in tumor growth dynamics. The majority of experimental (and theoretical) research on cell motion is carried out in two spatial dimensions, but it is becoming increasingly clear that movement in vivo, which of course is in three dimensions, is different in important ways. This paper develops a theoretical framework that allows, through a mechanical energy study, to determine conditions on the extracellular matrix (ECM) and cell properties, for single cells (or aggregates) to be able to invade ECM via squeezing through pores. They consider the implications of their results for metastasis.

Araujo et al. (2018) investigate metastasis in the context of prostrate cancer. Using a hybrid cellular automaton model in which signaling molecules are modeled via partial differential equations (PDEs), while cells are modeled as discrete entities, they explore possible scenarios in which prostrate cancer cell (PCa) clusters can successfully establish themselves. They show that such clusters must fall into a certain size range: While small clusters can recruit mesenchymal stem cells (MScs) to establish metastases, clusters that are too large can fail due to lack of resources. This work highlights the complex multiscale interplay involved between different cell types and key signaling molecules during bone metastatic prostrate cancer and suggests that targeting MScs could be a viable therapeutic strategy.

Adoptive immunotherapy is the subject of the paper by Talkington et al. (2017). It considers four ODE models from the literature that have been proposed to model adoptive immunotherapy in the context of acute lymphocytic leukemias. Analysis of the models, in some cases suitably modified, show that they can all predict that such therapy can clear, or reduce, tumors. However, the details of how treatment should be administered are shown to be different across these models, suggesting that care has to be taken in how the immune system is modeled. The study in this paper highlights a challenge for the field in general. Typically, groups work on developing their own mathematical models for a specific application. The reward system within academia encourages the development of new models and actually discourages comparative studies of different models, despite the fact that the latter is scientifically invaluable.

Continuing the immune system theme, Besse et al. (2017) present a 3-compartment ODE model for the immune response to chronic myelogenous leukemia (CML). This model, a simplification of their previously published model, is amenable to a very 
detailed mathematical analysis, and it is shown to fit patient data well. One of the conclusions of the model analysis is that relapse is possible after treatment cessation if certain conditions are met. The model thus has the potential to be used to determine which patients need to be closely monitored after treatment.

Different aspects of the tumor microenvironment are the theme of the next two papers. The paper by Friedman and Hao (2017) presents the first mathematical model that focuses on exosomes in the cancer microenvironment. The model comprises a large system of coupled PDEs with a free boundary and explores the interaction of tumor cells with the immune system. A detailed numerical simulation study is carried out, and it is shown that the model exhibits results consistent with experiments. Crucially, it also shows how the size of a tumor could be determined by measuring the expression of specific micro-RNAs, and a novel use of these as biomarkers for cancer is proposed.

Hoehme et al. (2018) extend a previously validated agent-based spatiotemporal model for hepatocyte-sinusoid alignment to investigate if this phenomenon is still active in early hepatocellular tumors. The model is simulated in realistic liver microarchitectures, and how the shape of the tumor depends on a number of key parameters, including tumor cell number, is explored. Model predictions on tumor architectures are validated experimentally. This work points out the potential importance of organ micro-architecture in understanding tumor phenotypes.

We then have five papers focusing on different forms of treatment: Burazin et al. (2017) develop a macroscopic model based on poroelasticity theory to describe the time evolution of a solid tumor and use it to investigate possible mechanisms responsible for the increased interstitial fluid pressure (IFP) observed in tumors when compared to healthy tissue. A conclusion is that the rise in tumor IFP is the same for different tumors, but occurs on different timescales. The model is numerically simulated to explore the effect of anti-angiogenic therapy, and it is found under which conditions this will be effective in lowering IFP.

Poleszczuk et al. (2017) address the problem of predicting tumor response to radiotherapy. The authors apply the linear-quadratic law to a number of ODE models for tumor growth and show that several models can fit data but give very different predictions on the outcome of therapy. They conclude that clinical recommendations should only follow when several different model predictions concur. This, once again, highlights the important fact that being consistent with the available data is just the first step in model "validation" and is, in fact, the starting point, not the end point, of any modeling study. One then needs to either show that model predictions are consistent across a suite of models before using the results clinically, or one needs to embark on a fully integrated study in which experiments/studies are designed specifically to continue the model validation process. Ideally, of course, one should do both.

Meanwhile, Lewin et al. (2018) present a new, spatially resolved model for the effects of radiation by extending the classical Greenspan (1972) model for tumor growth and including oxygen dependence. This leads to an integro-differential equation coupled to a reaction-diffusion equation. A combination of analysis and numerical simulation reveals a very rich behavior of this system, suggesting that the spatial heterogeneity arising through oxygen concentration may be important for predicting clinical response to radiation treatment. 
Williams et al. (2017) investigate additive damage models for combination of radiation and chemotherapy. The models are rational functions that predict the effects of damage to treatments, where the form of the functions encodes the hypotheses proposed for how damage leads to cell death. Detailed statistical analysis is carried out to compare and contrast models, and it is shown that the additive damage models outperform models assuming independent cell kill. Potential applications to optimization of treatment and analysis of new drug-radiation combinations are discussed.

Comparison of models is the theme of the paper by Swan et al. (2017), in the context of the spread of brain tumors. They consider their previously proposed PDE model that accounts for material anisotropy in the brain, thought to affect the ability of glioblastoma multiforme (GBM) cells to invade. Their model is first compared with other models, and then they use the model with patient-specific data to determine under which conditions the model captures the observed tumor growth sufficiently well to allow surgeons to make better informed decisions on what and where to excise.

Massey et al. (2017) consider a PDE model for glioma cell response to plateletderived growth factor (PDGF), where the cells are divided into PDGF-expressing retrovirally transduced cells and recruited (oligodendroglial progenitor) cells, extending their previous spatially one-dimensional model to two-dimensional anatomically accurate slices of brain, with spatially heterogeneous diffusion (determined by gray and white matter). They show that their mathematical model produces patterns of cell invasion that are consistent with those observed experimentally and that the tumor morphology depends critically on the different rates of migration of the cells. Their results also suggest that recruited cells lead to faster-growing tumors and this has therapeutic implications. For example, disrupting the paracrine signaling that leads to recruitment may potentially slow growth, leading to longer patient survival times.

We then have three papers on various aspects of network dynamics: Wynn et al. (2017) develop an approach to infer intercellular network circuitry from experimentally measured Western blot data using Boolean and genetic algorithm methods. The authors carry out a detailed evaluation of the approach and use the model to prioritize the next best experiment to perform to help determine the network. While their particular application is to epidermal growth factor signaling of a breast epithelial cell line, their approach is much more widely applicable.

Komarova and van den Driessche (2017) investigate control networks that exist between groups of cells in multicellular organisms, focusing in particular on homeostatic regulation of stem cell lineages. The authors formulate a general ODE model and look at the signs of the Jacobian matrix at steady state to determine stability, and this allows them to obtain general rules on how to build up stable minimal control networks and generate models that are irreducible.

Szymanska et al. (2017) use spatial stochastic models that are continuous-time, discrete-space Markov processes governed by a reaction-diffusion master equation. This paper considers two models - a stochastic spatiotemporal model for gene regulatory networks (NF-kappaB) and a force-based individual-based model for tumor cords. It presents a vision for how these models could be coupled (with more detailed pathway modeling) into a multiscale vascular tumor model that imports actual tumor images as initial conditions (e.g., the vasculature) and through the use of high-performance computing can simulate up to $10^{9}$ cells. 
Finally, the role of stem cells is explored in the paper by Yan et al. (2017). It presents a three-dimensional multiscale model of colon cancer organoids that incorporates different cell types and a number of key signaling molecules. It is shown that the model can capture many known experimental phenomena and confirm a number of hypotheses made on the role of stem cells in growth and morphology. In particular, the crucial role of feedback on the dynamics of the organoid morphology is shown and explored in detail.

Acknowledgements The authors would like to thank the Mathematical Biosciences Institute (MBI) at Ohio State University, for supporting the special emphasis year on Cancer and its Environment. MBI receives its funding through the National Science Foundation Grant DMS1440386.

\section{References}

Al Price, Spencer CCA, Donnelly P (2015) Progress and promise in understanding the genetic basis of common diseases. Proc R Soc B 282:20151684

Altrock PM, Liu LL, Michor F (2015) The mathematics of cancer: integrating quantitative models. Nat Rev Cancer 15(12):730-745

Anderson AR, Quaranta V (2008) Integrative mathematical oncology. Nat Rev Cancer 8(3):227-234

Araujo A, Cook M, Lynch CC, Basanta B (2018) Size matters: metastatic cluster size and stromal recruitment in the establishment of successful prostrate cancer to bone metastases. Bull Math Biol. https://doi.org/ 10.1007/s11538-018-0416-4

Basanta D, Anderson ARA (2017) Homeostasis Back and Forth: An Ecoevolutionary Perspective of Cancer. Cold Spring Harb Perspect Med. 7(9):a028332

Besse A, Clapp GD, Bernard S, Nicolini FE, Levy D, Lepoutre T (2017) Stability analysis of a model of interaction between the immune system and cancer cells in chronic myelogenous leukemia. Bull Math Biol. https://doi.org/10.1007/s11538-017-0272-7

Burazin A, Drapaca CS, Tenti G, Sivaloganathan S (2017) A poroelasticity theory approach to study the mechanisms leading to elevated interstitial fluid pressure in solid tumours. Bull Math Biol. https://doi. org/10.1007/s11538-017-0383-1

Burrell RA, McGranahan N, Bartek J, Swanton C (2013) The causes and consequences of genetic heterogeneity in cancer evolution. Nature 501(7467):338-345

Byrne HM (2010) Dissecting cancer through mathematics: from the cell to the animal model. Nat Rev Cancer 10(3):221-230

Egeblad M, Nakasone ES, Werb Z (2010) Tumors as organs: complex tissues that interface with the entire organism. Dev Cell 18(6):884-901

Friedl P, Alexander S (2011) Cancer invasion and the microenvironment: plasticity and reciprocity. Cell 147(5):992-1000

Friedman A, Hao W (2017) The role of exosomes in pancreatic cancer microenvironment. Bull Math Biol. https://doi.org/10.1007/s11538-017-0254-9

Gatenby RA, Gillies RJ (2008) A microenvironmental model of carcinogenesis. Nat Rev Cancer 8(1):56-61

Gatenby RA, Maini PK (2003) Mathematical oncology: cancer summed up. Nature 421(6921):321

Giverso C, Arduino A, Preziosi L (2017) How nucleus mechanics and ECM microstructure influence the invasion of single cells and multicellular aggregates. Bull Math Biol. https://doi.org/10.1007/s11538017-0262

Gravenmier CA, Siddique M, Gatenby RA (2017) Adaptation to stochastic temporal variations in intratumoral blood flow: the Warburg Effect as a bet-hedging strategy. Bull Math Biol. https://doi.org/10. 1007/s11538-017-0261-x

Greaves M, Maley CC (2012) Clonal evolution in cancer. Nature 481(7381):306-313

Greenspan H (1972) Models for the growth of a solid tumor by diffusion. Stud Appl Math 51:317-340

Hirata E, Girotti MR, Viros A, Hooper S, Spencer-Dene B, Matsuda M, Larkin J, Marais R, Sahai E (2015) Intravital imaging reveals how BRAF inhibition generates drug-tolerant microenvironments with high integrin $\beta 1$ /FAK signaling. Cancer Cell 27:574-588 
Hoehme S, Bertaux F, Weens W, Garsl-Kraupp B, Hengstler JG, Drasdo D (2018) Model prediction and validation of an order mechanism controlling the spatiotemporal phenotype of early hepatocellular carcinoma. Bull Math Biol. https://doi.org/10.1007/s11538-017-0375-1

Jain H, Jackson T (2017) Mathematical modelling of cellular cross-talk between endothelial and tumor cells highlights counterintuitive effects of VEGF-targeted therapies. Bull Math Biol. https://doi.org/ 10.1007/s11538-017-0273-6

Komarova NL, van den Driessche P (2017) Stability of control networks in autonomous homeostatic regulation of stem cell lineages. Bull Math Biol. https://doi.org/10.1007/s11538-017-0283-4

Lewin TD, Maini PK, Moros EG, Enderling H, Byrne HM (2018) The evolution of tumour composition during fractionated radiotherapy: implications for outcome. Bull Math Biol. https://doi.org/10.1007/ s11538-018-0391-9

Maley CC, Aktipis A, Graham TA, Sottoriva A, Boddy AM, Janiszewska M, Silva AS, Gerlinger M, Yuan Y, Pienta KJ, Anderson KS, Gatenby R, Swanton C, Posada D, Wu C-I, Schiffman JD, Hwang ES, Polyak K, Anderson ARA, Brown JS, Greaves M, Shibata D (2017) Classifying the evolutionary and ecological features of neoplasms. Nat Rev Cancer 17:605-619

Marusyk A, Almendro V, Polyak K (2012) Intra-tumour heterogeneity: a looking glass for cancer? Nat Rev Cancer 12(5):323-334

Massey SC, Rochne RC, Hawkins-Daarud A, Gallaher J, Anderson ARA, Canoll P, Swanson KR (2017) Simulating PDGF-driven glioma growth and invasion in an anatomically accurate brain domain. Bull Math Biol. https://doi.org/10.1007/s11538-017-0312-3

Meacham CE, Morrison SJ (2013) Tumour heterogeneity and cancer cell plasticity. Nature 501(7467):328-337

Meads MB, Gatenby RA, Dalton WS (2009) Environment-mediated drug resistance: a major contributor to minimal residual disease. Nat Rev Cancer 9(9):665-674

Poleszczuk J, Walker R, Moros EG, Latifi K, Candell JJ, Enderling H (2017) Predicting patient-specific radiotherapy protocols based on mathematical model choice for proliferation saturation index. Bull Math Biol. https://doi.org/10.1007/s11538-017-0279-0

Robertson-Tessi M, Gillies RJ, Gatenby RA, Anderson AR (2015) Impact of metabolic heterogeneity on tumor growth, invasion, and treatment outcomes. Cancer Res 75(8):1567-1579

Swan A, Hillen T, Bowman JC, Murtha AD (2017) A patient-specific anisotropic diffusion model for brain tumour spread. Bull Math Biol. https://doi.org/10.1007/s11538-017-0271-8

Swanton C (2012) Intratumor heterogeneity: evolution through space and time. Cancer Res 72(19):4875-4882

Szymanska Z, Cytowski M, Mitchell E, Macnamara CK, Chaplain MAJ (2017) Computational modelling of cancer development and growth: modelling at multiple scales and multiscale modelling. Bull Math Biol. https://doi.org/10.1007/s11538-017-0292-3

Talkington A, Dantoin C, Durrett R (2017) Ordinary differential equation models for adoptive immunotherapy. Bull Math Biol. https://doi.org/10.1007/s11538-017-0263-8

Tredan O, Galmarini CM, Patel K, Tannock IF (2007) Drug resistance and the solid tumor microenvironment. J Natl Cancer Inst 99(19):1441-1454

Turner NC, Reis-Filho JS (2012) Genetic heterogeneity and cancer drug resistance. Lancet Oncol 13(4):e178-e185

Williams KS, Secomb W, El-Kareh AW (2017) Additive damage models for cellular pharmacodynamics of radiation-chemotherapy combinations. Bull Math Biol. https://doi.org/10.1007/s11538-017-0316-Z

Wynn ML, Egbert M, Consul N, Chang J, Wu Z-F, Meravjer SD, Schnell S (2017) Inferring intracellular signal transduction circuitry from molecular perturbation experiments. Bull Math Biol. https://doi. org/10.1007/s11538-017-0270-9

Yan H, Konstorum A, Lowengrub JS (2017) Three-dimensional spatiotemporal modeling of colon cancer organoids reveals that multimodal control of stem cell self-renewal is a critical determinant of size and shape in early stages of tumor growth. Bull Math Biol. https://doi.org/10.1007/s11538-017-0294-1 